\title{
La falta de homogeneidad del producto (FHP) en las empresas cerámicas y su impacto en la reasignación del inventario
}

\author{
F. ALARCÓN, M.M.E. ALEMANY, F.C. LARIO, R.F. OLTRA
}

Centro de Investigación en Gestión e Ingeniería de la Producción (CIGIP), Universidad Politécnica de Valencia, Valencia, España

\begin{abstract}
La asignación del producto disponible a prometer (ATP) a pedidos en contextos de fabricación contra almacén (MTS) es de la máxima importancia ya que puede influir en la satisfacción del cliente y en los beneficios de la empresa. Sin embargo, una asignación inicial adecuada, puede pasar a ser inadecuada por diversas razones. En estos casos, es necesaria la reasignación del inventario, la cual será más compleja cuanto más ambiciosos sean los objetivos a alcanzar con ella y mayor el volumen de información a utilizar. En este sentido, cabe destacar que la falta de homogeneidad en el producto (FHP), presente en distintos sectores industriales, provoca la atomización del inventario y aumenta la complejidad de la reasignación, dificultando la obtención de soluciones óptimas. En el presente trabajo se describe la problemática de la FHP, primero de manera genérica, y luego, particularizada a empresas cerámicas MTS. Posteriormente, se identifican las situaciones en las que una determinada asignación de ATP puede dejar de ser adecuada en dicho contexto y se propone la reasignación como una forma de búsqueda de nuevas asignaciones válidas. Finalmente, mediante un caso de estudio de una empresa cerámica, se analiza el impacto de la FHP en cada una de las situaciones identificadas, observando que la FHP provoca alguna de éstas situaciones y complica, en todas ellas, la reasignación del inventario a pedidos.
\end{abstract}

Palabras clave: Asignación de inventario, disponible a prometer (ATP), falta de homogeneidad en el producto (FHP), fabricación contra stock (MTS)

The lack of homogeneity in the product (LHP) in the ceramic tile industry and its impact on the reallocation of inventories

The allocation of the product available- to-promise (ATP) in make-to-stock (MTS) contexts is of the utmost importance as it can influence customer satisfaction and profits of the company. However, a proper initial allocation may become inadequate for several reasons. In these case, it is necessary the reallocation of inventory, which will be more complex the more ambitious goals to achieve with it and increased the amount of information to use. In this regard, it is noteworthy that the lack of homogeneity in the product (LHP), present in different industrial sectors, causes the atomization of the inventory and increases the complexity of the reallocation, difficult to obtain optimal solutions. This paper describes the problems of the LHP, first under a generic perspective and then, particularized to MTS ceramic companies. Subsequently, situations in which a specific allocation of ATP can no longer be appropriate in this context are identified and the reassignment, as a way to search for new valid assignments, is proposed. Finally, through a case study of a ceramic company, the impact of the LHP in each of the situations identified is analyzed, noting that the LHP causes some of these situations and in all of them, complicates the reallocation of inventory to orders.

Keywords: stock allocation, available to promise (ATP), lack of homogeneity in the product (LHP), make to stock (MTS)

\section{INTRODUCCIÓN}

En la fabricación contra stock ("Make to Stock" ó MTS), algunos de los procesos de negocio fundamentales son pronosticar la demanda, planificar la fabricación, fabricar, almacenar el producto y comprometerse con el cliente para atender sus propuestas de pedido. En el proceso de comprometer pedidos es fundamental el uso del concepto de disponible a prometer o ATP ("Available To Promise"). El ATP puede definirse como la cantidad disponible, real o planificada, de producto final no comprometida con ningún cliente (1). El ATP se irá comprometiendo con el cliente mediante el proceso de comprometer pedidos, definido como el conjunto de actividades que se realizan para analizar en qué medida es posible comprometerse con el cliente sobre la propuesta de pedido que éste entrega a la empresa (2).
Una adecuada asignación de ATP a las propuestas de pedido es fundamental para conseguir un elevado nivel de satisfacción del cliente y para incrementar los beneficios de la empresa. Sin embargo, una asignación adecuada u óptima en un momento dado, puede pasar a ser no adecuada, no óptima, o incluso infactible, en función de posibles acontecimientos imprevistos como, por ejemplo, un retraso en la llegada de materia prima (3) o la llegada de nuevos pedidos más urgentes que los ya comprometidos (los cuales requieren productos ya reservados). En estos casos, puede ser interesante realizar una reasignación de inventario, tanto del real como del correspondiente a los lotes de producción planificados, a todos los pedidos ya comprometidos y a los nuevos que se pretendan comprometer, con el objetivo de mejorar el 
nivel de servicio al cliente, al tiempo que se incrementen los beneficios de la empresa. Pero la reasignación puede llegar a ser complicada, especialmente cuando se debe manejar un gran volumen de información y los objetivos perseguidos son muy ambiciosos.

Por otra parte, existen algunos sectores industriales como el cerámico, hortofrutícola, marmolero, maderero, textil o cárnico, en los que se obtienen productos que, debiendo ser iguales, presentan alguna pequeña diferencia en alguna de sus cualidades, lo cual complica la reasignación de inventario. Esto puede ocurrir cuando la materia prima utilizada para la fabricación de dichos productos proviene directamente de la naturaleza (4). También cuando alguna de las características del producto depende de factores que no se puedan controlar durante su proceso de fabricación o en un momento determinado (presión, humedad, temperatura, etc.), como ocurre en muchos procesos de transformación multietapa, en los que la variación observable en una determinada característica del producto es consecuencia de la generada y transmitida por las distintas etapas del proceso (5). La existencia de estas pequeñas diferencias conlleva una Falta de Homogeneidad en el Producto (FHP) y, cuando los pedidos de los clientes deben servirse con producto homogéneo, obliga a una clasificación en subtipos de producto homogéneo. Esta necesaria clasificación provoca una atomización del inventario debido a la constante aparición de nuevos subtipos de productos que multiplican el número de posibles combinaciones a la hora de servir pedidos, así como el volumen de información a utilizar, complicando, por lo tanto, la reasignación del inventario.

En este contexto, la FHP se podría definir como "la carencia de la homogeneidad requerida por el cliente en los productos" y se debe a la imposibilidad de fabricar productos homogéneos, bien en un mismo lote o entre los distintos lotes obtenidos de un proceso. La FHP supone un problema cuando el cliente de dicho proceso adquiere varias unidades de un lote o de distintos lotes de producto y requiere homogeneidad en ellas porque se han de utilizar, presentar, colocar o consumir conjuntamente.

La problemática de la FHP puede abordarse desde dos perspectivas: a) desde una perspectiva tecnológica centrada en la reducción o eliminación de las causas de la FHP, mediante la cual se tratan de modificar y mejorar los procesos de fabricación, las características de los productos y las características de las materias primas, y b) desde una perspectiva de Dirección de Operaciones, que asume la existencia de la FHP y trata de reducir su impacto en el cliente y en los costes.

En este sentido, el principal objetivo del presente trabajo consiste en mostrar cómo, desde la perspectiva de la Dirección de Operaciones, las decisiones relativas a la asignación y reasignación de inventario son fundamentales para alcanzar la necesaria flexibilidad que permita el cumplimiento de los compromisos adquiridos con el cliente de la manera más eficiente para la empresa, especialmente en contextos en los que existe FHP. Aunque el análisis realizado se concreta para empresas del sector cerámico, es necesario resaltar que la conexión de la reasignación de inventario con la FHP abre una nueva línea de investigación que está siendo aprovechada por los investigadores para mejorar la gestión del inventario y la satisfacción del cliente, reducir costes y aumentar los ingresos en empresas de otros sectores.

Una vez definido y formalizado el concepto de FHP, se pasa a describir cómo se encuentra estructurado el resto del trabajo. En el apartado 2, se lleva a cabo una revisión de la literatura relevante sobre la asignación de ATP a pedidos: En el apartado 3, se describe la problemática de la FHP en empresas cerámicas con enfoque MTS. Posteriormente, en la sección 4, a través de un caso de estudio de una empresa líder del sector cerámico español, se identifican las situaciones en las que una determinada asignación de ATP a pedidos puede dejar de ser adecuada y se propone la reasignación como una forma de búsqueda de nuevas asignaciones válidas. A continuación, se analiza el impacto de la FHP en cada una de las situaciones identificadas, observando que la FHP provoca una de éstas situaciones y complica, en todas ellas, la reasignación del inventario a pedidos. Para cada una de las situaciones de reasignación analizadas, se incluye un ejemplo numérico extraído de la realidad, aunque simplificado, que permite mostrar cómo se produce la correspondiente situación y cómo influye la FHP en ella.

\section{REVISIÓN DE LA LITERATURA}

Una manera ampliamente aceptada de clasificar los modelos que tratan de asignar ATP a pedidos de clientes se basa en el tipo de respuesta o modo de funcionamiento de dichos modelos (6-8). Desde este punto de vista, algunos modelos tratan de asignar el ATP en tiempo real ("on-line" o "realtime") para proporcionar una respuesta inmediata a cada propuesta de pedido, según ésta llega y el orden en que llega ("first come first served" ó FCFS) (9-11). Otros modelos de asignación de ATP, sin embargo, utilizan un modo de respuesta diferido ("off-line"), agrupando las propuestas de pedido que llegan durante un intervalo de tiempo prefijado ("batch interval") en lotes ("batch") (6) y proporcionando soluciones para cada lote de propuestas $(9,12,13)$. Está comprobado que éstos últimos proporcionan mejores resultados que los que funcionan en tiempo real (6).

Sin embargo, una determinada asignación, aún siendo adecuada u óptima en un momento dado, puede pasar a ser no adecuada u óptima o incluso infactible, en función de posibles acontecimientos imprevistos. En esta línea, M. O. Ball et al. (6) sostienen que una determinada asignación puede dar una visión "miope" de la situación ya que, a los pedidos que van llegando, se les asigna producto sin tener en cuenta el impacto que ello tendrá en la cumplimentación de los pedidos que llegarán en un futuro. Esto mismo se corrobora por R. Pibernik y P. Yadav (8), quienes explican el problema de servir pedidos en un sistema en el que hay una desviación importante entre la previsión de demanda y la demanda real, lo que origina situaciones, tanto de exceso como de escasez de inventario.

Esta visión miope puede mitigarse, hasta cierto punto, con una política de "racionamiento de inventario" (8). Mediante el concepto de racionamiento de inventario, ya utilizado por D. M. Topkis (14), se considera la asignación del inventario, previamente clasificado según diferentes clases de demanda. Posteriormente, S. Benjaafar et al. (15) tienen en cuenta diferentes niveles de servicio y costes de retraso asociados a los tipos de cliente, y establecen un umbral para cada clase de cliente, siendo óptimo satisfacer la demanda de una clase de clientes siempre que el inventario físico ("on hand") esté por encima del umbral establecido. En este último trabajo citado se determina que, el racionamiento de inventario en 
sistemas con múltiples clases de clientes, es superior a la política de asignación de inventario en la que se sirven los pedidos en orden FCFS. En esta línea, otros trabajos presentan modelos para la asignación de ATP en situaciones de escasez de inventario, con diferentes políticas de asignación (FCFS, "Rank-based", "optimization-based", y "preallocation") (16), o incluyen modelos deterministas para la asignación de ATP a diferentes clases de clientes (17).

Pero con políticas de racionamiento de inventario no se puede asegurar la obtención de asignaciones óptimas de ATP porque se definen en base a una estimación del tipo y cantidad de demanda que se espera recibir. Esto queda parcialmente resuelto en los modelos que procesan lotes de propuestas de pedido en modo diferido y que, por el contrario, no proporcionan respuestas a los clientes hasta que no haya transcurrido el intervalo de tiempo fijado. Dichos modelos, por otra parte, no aseguran que se puedan comprometer todas las propuestas de pedido recibidas en cada intervalo de tiempo.

En ciertos casos, puede ser recomendable complementar un método de respuesta en tiempo real con una posterior ejecución en diferido que reasigne el inventario a los pedidos ya comprometidos con el objetivo de paliar, en cierta medida, el carácter miope del método en tiempo real. En este sentido, un posible modo de funcionamiento en diferido es la "reasignación de inventario" a la cartera de pedidos ya comprometidos. La reasignación de inventario consiste en volver a decidir a qué grupo de pedidos y de qué forma se asigna el inventario, inicialmente asignado a un determinado grupo de pedidos (posiblemente, con el modo de respuesta en tiempo real). La reasignación de inventario podría utilizarse, por lo tanto, para mejorar la solución obtenida por el modo de respuesta en tiempo real. Es más, debido a diferentes fuentes de incertidumbre (comentadas en la sección 3), existen situaciones en las que la reasignación del inventario, no sólo mejora una asignación previa, sino que es la única alternativa para obtener una solución factible.

Pese a la importancia de una adecuada reasignación de reservas para garantizar que cada pedido se sirve en la fecha comprometida, aumentar la satisfacción del cliente, mejorar la gestión del stock, reducir costes y aumentar los ingresos de la empresa, la bibliográfica consultada ha permitido verificar que la mayoría de los trabajos sobre sistemas ATP se centran en la asignación de ATP y no identifican claramente las situaciones en las que se hace necesaria una reasignación ni abordan dicho asunto. A este respecto, cabe destacar que la línea de trabajo más próxima al concepto de reasignación de inventario es la de los modelos de respuesta en modo diferido, aunque no se ha encontrado ningún trabajo que relacione la problemática de la FHP con la asignación de ATP, ni siquiera que estudie la FHP desde la perspectiva de la Dirección de Operaciones.

\section{LA PROBLEMÁTICA DE LA FHP EN LAS EMPRESAS CERÁMICAS CON ENFOQUE MTS}

La FHP es característica en las empresas de fabricación de productos cerámicos, donde diferentes factores relacionados con el proceso productivo y las materias primas $(18,19)$, como la humedad $(20,21)$, la temperatura (22), o el origen y propiedades de las arcillas y pigmentos (23), afectan de forma impredecible e inevitable al aspecto final del producto. En este sentido, E. Vallada et al. (24) afirman que, aparte de posibles defectos, el proceso de producción, y sobre todo los ciclos de producción cortos, generan falta de uniformidad en los colores (diversidad de tonos) y en los tamaños (diversidad de calibres). También en esta línea, J. A. Heredia y M. Gras (5) sostienen que la variación dimensional en los procesos de fabricación de baldosas cerámicas es un problema todavía no resuelto y, pese a los esfuerzos realizados por los fabricantes para controlar el proceso, los resultados no cumplen con las especificaciones del mercado. Precisamente, según A. Poyatos et al. (25), la dificultad para la consecución de lotes de producción con piezas de un mismo tamaño o calibre es uno de los problemas más importantes del sector de fabricación de baldosas cerámicas.

Por ello, una de las fases del proceso de fabricación cerámico, antes del paletizado y empaquetado, es la clasificación del producto obtenido (26). Según J. A. Heredia y M. Gras (5), la clasificación del producto acabado en subgrupos de diferentes dimensiones es obligatoria para los fabricantes debido a que la variación dimensional observable en el producto es superior a las tolerancias que establece el mercado. Esta fase de clasificación se realiza, básicamente, de forma manual (27), pese a los intentos de automatizarla mediante diferentes tecnologías o herramientas, como la visión artificial (28-31), centradas en la discriminación del color o tonalidad.

En esta fase de clasificación, el lote fabricado se atomiza, es decir; un lote de un determinado tamaño, en el que tono y calibre deberían ser exactamente iguales para cada producto obtenido, pasará a descomponerse en dos o más sublotes de menor tamaño, según las diferentes combinaciones de tono y calibre realmente obtenidas. En realidad, la clasificación del producto final se realiza en la actualidad atendiendo a cuatro criterios (25): aspecto superficial, planaridad, tonalidad y calibre. Si el aspecto superficial o la planaridad de las piezas no alcanzan los requisitos mínimos exigidos por la empresa, el producto se desecha directamente. Los productos que superan esta criba se clasifican normalmente, en función de su aspecto superficial, en las categorías: primera, segunda o tipo único. Para las dos primeras categorías (primera y segunda) el producto se clasifica según su tonalidad o tono (normalmente dos clases) y según su tamaño o calibre (normalmente tres clases). De esta forma, para un modelo concreto de producto, pueden encontrarse trece referencias distintas.

A partir de la clasificación, cada nuevo sublote, con una combinación de calidad, tono y calibre deberá ser identificado, almacenado y gestionado por separado. En este contexto, es importante tener en cuenta que, en los sistemas MTS, debido a razones tecnológicas y de eficiencia en producción, los lotes lanzados a fabricar son mayores que la demanda comprometida en el momento del lanzamiento, creándose así un inventario que se deberá gestionar de manera adecuada para servir posteriores pedidos.

La gestión adecuada de los distintos sublotes obtenidos será decisiva para la satisfacción del cliente. Lo importante en este sentido será servir pedidos con productos de una única calidad, tono y calibre, evitando que a un cliente le lleguen productos con distintas características y así, los consiguientes problemas estéticos y funcionales: pavimentos y revestimientos cerámicos de un mismo color, pero con distintos tonos, que empeoran el acabado del conjunto de la pared o suelo, o diferentes calibres, que provocan desniveles o "escalones" en un mismo suelo o pared. 
En las empresas cerámicas, la FHP empeora el aspecto del conjunto de productos adquiridos y cobra especial relevancia, por ejemplo, cuando hay que realizar reposiciones, reparaciones o sustituciones de partes de un conjunto anteriormente adquirido por el cliente. En estos casos no se le pueden servir al cliente productos con falta de homogeneidad respecto a los que ya tiene, lo que añade una nueva restricción a la problemática de la reasignación del inventario.

En definitiva, la FHP aumenta la complejidad en las empresas cerámicas, tanto de la asignación de ATP como de la reasignación del inventario, debido al efecto de la atomización del inventario que: aumenta las posiciones necesarias en el almacén y complica su gestión, incrementa el volumen de información a manejar y las posibles combinaciones para servir los pedidos, crea la necesidad de sobredimensionar los lotes de fabricación e impone nuevas restricciones en la toma de decisiones.

Cabe destacar que, según se pone de manifiesto en la revisión de la literatura, los trabajos consultados tratan la problemática de la FHP desde una perspectiva tecnológica. Los autores no tienen constancia de trabajos que analicen y propongan soluciones desde una perspectiva de Dirección o Gestión de Operaciones siendo por ello, dicho enfoque, una de las principales aportaciones del presente trabajo. Actualmente, se da mucha importancia al diseño de sistemas de planificación y control de operaciones flexibles, capaces de adaptarse a un entorno cada vez más turbulento. Sin embargo, dichos sistemas, tratan de dar respuesta a la incertidumbre proveniente del entorno, como por ejemplo, la de la demanda de los clientes. Son más escasos los trabajos que incorporan la incertidumbre en el proceso productivo, y estos lo hacen fundamentalmente en relación a los tiempos de suministro. La FHP introduce un nuevo tipo de incertidumbre: la incertidumbre en las cantidades de producto homogéneo disponible. Esta incertidumbre debe considerarse cuando el cliente requiere unidades homogéneas de un mismo producto. En estos casos, la conexión entre los sistemas de planificación y control de operaciones y los sistemas ATP, debe ser muy estrecha, de manera que se pueda dotar al sistema de gestión de la suficiente flexibilidad para adaptarse a esta fuente adicional de incertidumbre, mientras no sea posible eliminarla definitivamente desde la perspectiva tecnológica.

A continuación, una vez explicadas las implicaciones de la FHP en el ámbito de empresas cerámicas MTS, se identifican y describen las situaciones en las que es necesaria la reasignación de inventario y se explica cómo la FHP impacta en cada una de ellas.

\section{LA REASIGNACIÓN DEL INVENTARIO EN EMPRE- SAS CERÁMICAS CON FHP Y ENFOQUE MTS. UN CASO DE ESTUDIO.}

La empresa, en la que se identifica la problemática objeto de estudio, pertenece a un grupo industrial español emplazado en la provincia de Castellón dedicado, desde mediados de los años setenta, al diseño, fabricación, comercialización y distribución de: pavimentos y revestimientos cerámicos tradicionales de pasta blanca y roja, sofisticados revestimientos pulidos y rectificados, porcelánicos técnicos y esmaltados de altas cualidades técnicas. En esta empresa, con la que los autores han llevado a cabo diferentes proyectos de investigación durante los últimos años, se atienden pedidos de millones de clientes situados en 150 países diferentes con un equipo humano de 600 personas. Sus instalaciones aseguran un suministro de calidad a todos sus clientes con una capacidad instalada superior a los 11 millones de metros cuadrados anuales.

Con objeto de mejorar la satisfacción del cliente, la empresa persigue proporcionar una respuesta lo más rápida y fiable posible a las propuestas de pedido del cliente (1). Por esta razón, el proceso mediante el cual se comprometen los pedidos, se ejecuta cada vez que llega una propuesta de pedido de un cliente y la respuesta se da en tiempo real, de manera que la fiabilidad de cumplir posteriormente con el compromiso adquirido con el cliente pueda ser máxima.

Adicionalmente, las empresas cerámicas con FHP en general y, la empresa cerámica objeto de estudio en particular, en donde el inventario está atomizado, deberán asegurarse mediante una asignación adecuada del ATP a pedidos de que a un mismo cliente no le llega producto heterogéneo. Pero, una determinada asignación inicial puede dejar de ser adecuada cuando las condiciones en las que se realizó dicha asignación cambian, en cuyo caso se hará necesaria una reasignación de inventario a pedidos.

Se han identificado tres situaciones que pueden convertir una asignación inicial adecuada u óptima en no adecuada, no óptima o incluso infactible $y$, por lo tanto, justificar la reasignación de inventario: 1) existencia de discrepancias entre lo planificado y lo obtenido realmente en fabricación, 2) llegada de nuevos pedidos prioritarios y 3) modificaciones por parte del cliente en las características de los pedidos ya comprometidos. Las tres situaciones anteriores pueden darse en cualquier sector productivo, pero en caso de sectores con FHP como el sector cerámico, la incertidumbre en las cantidades homogéneas disponibles de producto tras el proceso de fabricación asegura que siempre van a existir discrepancias entre lo planificado y lo realmente obtenido. Por otro lado, en las tres situaciones mencionadas, la existencia de disponibilidades de varios subtipos de un mismo producto para poder servir el pedido de un cliente, incrementa sustancialmente tanto las posibilidades de servir un pedido, como la cantidad de información a manejar y complica la obtención de soluciones no ya optimas sino, lo que es más importante, factibles.

Para explicar la manera en la que la FHP impacta en cada una de estas tres situaciones y justificar la necesidad de reasignar el inventario, se han utilizado datos históricos basados en pedidos, inventario y planes de producción reales de la empresa citada. A este respecto, cabe indicar que la cartera de pedidos analizada en el momento de nuestro estudio, consta de más de 2.000 pedidos de los cuales, el 50\% tienen más de una línea de pedido. Además, en la empresa se manejan más de 100 referencias que, por el efecto de atomización debido a la FHP, se convierten en miles de referencias distintas en almacén. El horizonte de tiempo para el que se reservan los pedidos llega a comprender más de 90 días. Por todo ello, la evolución del ATP en esta empresa a lo largo del tiempo es constante y compleja, y un estudio exhaustivo requeriría el manejo de un gran volumen de información, adquiriría unas dimensiones desproporcionadas y no facilitaría el entendimiento de la problemática abordada. 
Por todo ello y por razones de confidencialidad, los datos inicialmente facilitados por la empresa han sido convenientemente adaptados y simplificados para el presente trabajo. De esta forma, para la descripción de cada situación, se considera sólo un horizonte de reasignación de 6 días (periodos) y se reproduce la llegada de propuestas de pedido codificadas como Pi $(i=1,2,3 \ldots$.$) , donde i indica el orden de$ llegada de cada propuesta a la empresa.

En la primera de las tres situaciones, se muestra la evolución del ATP, tanto real como planificado, a lo largo de los 6 días considerados. Cada propuesta de pedido, en el caso de que se pueda servir, se convierte en pedido en firme y se coloca en el día o periodo correspondiente a su fecha de entrega, actualizando el ATP. En la segunda y tercera de las situaciones se presenta la evolución del ATP real según van llegando diferentes propuestas de pedido.

En la empresa considerada, cada propuesta de pedido contiene la siguiente información, que se utiliza total o parcialmente, según proceda en cada situación: código o número de pedido $(\mathrm{Pi})$, fecha de entrega, importe y, para cada línea de pedido, cantidad de producto solicitado. Según la política comercial de la empresa, las propuestas de pedido se deberán servir completas es decir, no se admitirán entregas parciales para ninguna de sus líneas. Tanto las cantidades de ATP como las solicitadas en las propuestas de pedido vienen expresadas en $\mathrm{m}^{2}$.

Un mismo producto puede provenir de distintos lotes de fabricación y, por el efecto de la FHP, tener distintas calidades, tonos y calibres. Para reflejar este hecho, en la empresa se codifican los productos, en función de la calidad, tono, calibre y lote del que provienen. Por ejemplo, $A 1^{\mathrm{a}} \mathrm{T} 1 \mathrm{C} 1 \mathrm{~L} 10$ hace alusión al producto $\mathrm{A}$, de $1^{\mathrm{a}}$ calidad, Tono 1 , Calibre 1 , y que proviene del lote de fabricación 10.

Para simplificar y adecuar la exposición del problema se ha considerado un sólo tipo de producto (modelo"A") con una única calidad y con dos posibles valores para tono y calibre, respectivamente. Así, en función de las diferentes combinaciones de tonos y calibres que se pueden dar, es posible encontrar cuatro subtipos de este producto A. Debido a esta simplificación, el código será siempre igual en sus dos primeras posiciones por lo que éstas son innecesarias. Así, el código a utilizar en el resto del trabajo sólo necesita reflejar el tono, el calibre y el lote (por ejemplo T1C1L10).

La empresa objeto de estudio realiza una asignación de ATP a las propuestas de pedido en tiempo real, según una política FCFS. Esta política es la más ampliamente aceptada en modelos de arrastre o "pull" según (6) y es empleada en la mayor parte de sistemas de gestión y ERP's del mercado (SAP, Axapta, Navision, Baan, CCS, etc.) mediante la opción de "reserva automática". Adicionalmente, esta política es frecuentemente utilizada en las empresas cerámicas, cuyas redes de tiendas o comerciales atienden los pedidos uno a uno y además en tiempo real, tratando de adquirir el compromiso con el cliente en el momento en el que éste realiza su propuesta de pedido, en vez de agrupar las propuestas en lotes y tratarlas con posterioridad, en modo diferido (6).

En cada propuesta de pedido, el cliente demanda una cierta cantidad de un tipo de producto (según se ha indicado, en este trabajo sólo se considera el modelo " $\mathrm{A}^{\prime \prime}$ ) para una determinada fecha de entrega, sin especificar ni calidad, ni tono, ni calibre, siendo en el proceso de asignación donde se decidirá calidad, tono y calibre específicos.

\subsection{Impacto de la FHP en la reasignación del inventario cuando hay discrepancias entre lo planificado y lo obtenido realmente en fabricación:}

La FHP tiene una incidencia clara en las reservas realizadas en base al ATP planificado, ya que genera un grado de incertidumbre elevado en cuanto a los tonos y calibres que finalmente se obtendrán en fabricación, pese a lo que se haya planificado. Por ello, una vez terminada la fabricación de un lote, y realizada la clasificación de dicho lote en los correspondientes sublotes según los tonos y calibres de los productos obtenidos, habrá que comprobar que se pueden servir los pedidos que habían reservado productos del lote planificado antes de fabricarse. El efecto de atomización del lote provocado por la FHP puede hacer que, pedidos que inicialmente han reservado de un lote, no puedan servirse porque no se haya obtenido ningún sublote lo suficientemente grande como para cubrir dichos pedidos. En este sentido, la incertidumbre inherente a la FHP puede hacer necesaria una reasignación de pedidos cada vez que finalice la fabricación de un lote.

Es importante destacar que la FHP impide que se puedan acumular o agrupar las distintas cantidades de ATP provenientes de distintos lotes planificados, bien en el mismo o en distinto periodo de tiempo, como se suele hacer en contextos sin FHP. La razón de ello es que, los lotes con FHP, estarán compuestos de productos de características distintas en cuanto a tono y calibre, y desconocidas hasta el momento de finalizar la fabricación. Esto puede provocar situaciones ilógicas, como la de tener que reservar ATP real, y no planificado, para un pedido de fecha de entrega tardía, a pesar de haber restos de ATP planificado cercanos a su fecha de entrega que se podrían acumular, si no hubiera FHP, y ser suficientes para servirlo.

A continuación se incluye una tabla (Tabla I), en la que se muestra la evolución del ATP a lo largo de los seis periodos considerados y la situación del ATP después de la asignación. Las filas de la tabla están organizadas en tres bloques. En el primero se incluye información sobre el ATP, tanto real ( $1^{\mathrm{a}}$ fila) como planificado ( $2^{\text {a }}$ fila). El ATP real reflejado en el periodo 1 está formado por productos con tono y calibre concretos que provienen de lotes fabricados antes del periodo 1. En la fila de ATP planificado se incluye la cantidad planificada a fabricar de producto en cada periodo, pero todavía no se especifica tono ni calibre porque no se conocen aún. En el segundo bloque se encuentra la información sobre pedidos. Esta incluye el código del pedido ( $3^{\mathrm{a}}$ fila), la cantidad en $\mathrm{m}^{2}$ solicitada por el cliente en cada pedido ( $4^{a}$ fila) y de dónde se reserva la cantidad solicitada en cada pedido ( $5^{\text {a }}$ fila). En el tercer bloque se incluye la información relativa al ATP que queda en cada periodo después de asignar producto a los pedidos que van llegando, diferenciando entre ATP real $\left(6^{\mathrm{a}}\right.$ fila) y ATP planificado ( $7^{\mathrm{a}}$ fila).

Las propuestas de pedido están situadas en los periodos correspondientes a sus fechas de entrega y su número indica el orden de llegada. El primer pedido que llega es el P1 y su fecha de entrega se corresponde con el periodo 5, por lo que dicho pedido se sitúa en la columna 5 . Según la política de la empresa, se intentará primero asignar producto para este pedido de algún lote planificado en la fecha de entrega del pedido (dejando así la mayor cantidad posible de ATP real para propuestas urgentes que lleguen a última hora). En el caso de no haber suficiente ATP en la fecha de entrega del pedido se buscará, por orden de cercanía, en 
los lotes de ATP planificados inmediatamente anteriores a la fecha de entrega. Si tampoco hay suficiente, se recurrirá, por último, al ATP real. En el caso de no haber ATP, la propuesta de pedido será rechazada y, en el caso de haberlo, se realizará la consiguiente asignación de ATP y reserva. Cuando en un periodo haya ATP proveniente de varios sublotes para asignar a una propuesta de pedido, se elegirá siempre la opción que, descontada la cantidad solicitada, deje el menor resto de sublote posible de producto.

Según se puede observar en la Tabla I, el lote L70 de $1600 \mathrm{~m}^{2}$ está planificado para el mismo periodo 5 así que, como tiene cantidad suficiente, se asignará producto de éste al pedido P1. Por ello, en la $5^{\text {a }}$ fila (donde se indica de dónde se reserva el producto) se colocará L70. Al hacer esta asignación se debe actualizar al ATP planificado después de asignación, así que, en la $7^{\text {a }}$ fila de la tabla I, se restarán los $\mathrm{m}^{2}$ del pedido $\mathrm{P} 1$ al lote planificado, de manera que ahora, el ATP planificado restante para ese periodo será de 1600-72 $\mathrm{m}^{2}$. El segundo pedido P2 debe entregarse el día o periodo 3 así que se coloca en la $3^{\mathrm{a}}$ columna. El primer lote planificado anterior a la fecha de entrega de P2 es el lote L50. Como L50 tiene cantidad suficiente de producto $\left(1510 \mathrm{~m}^{2}\right)$, se asignará el correspondiente producto de dicho lote a P2, por lo que en la $5^{\mathrm{a}}$ columna se colocará el valor de L50 y en la $7^{\mathrm{a}}$ fila se actualizará la cantidad de ATP planificado después de asignación, que pasará a ser de 1510-515=995 $\mathrm{m}^{2}$.

TABLA I. ASIGNACIÓN DEL ATP REAL Y PLANIFICADO A LAS CINCO PROPUESTAS DE PEDIDO CONSIDERADAS.

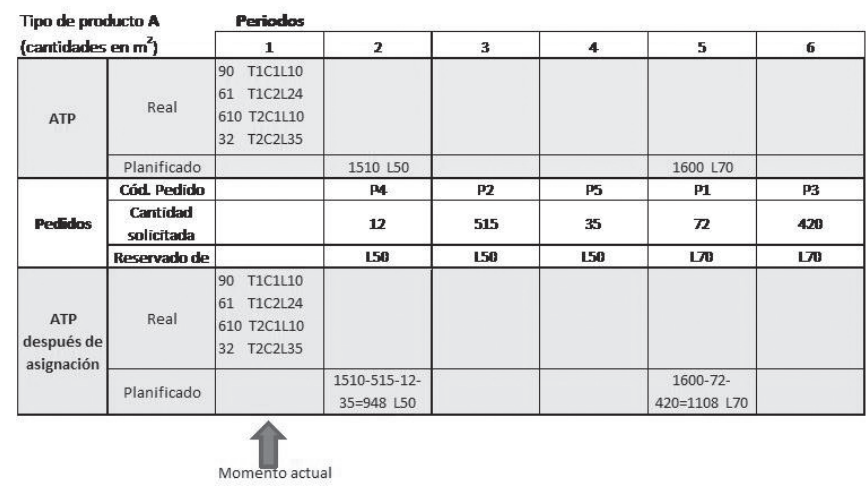

Como se puede observar en la Tabla I, el ATP real inicial en el periodo 1 no es una única cantidad, como ocurriría en una empresa sin FHP, sino cantidades con tonos y calibres diferentes que provienen de distintos lotes de fabricación (T1C1L10, T1C2L24, ...). Precisamente, estos son los restos que se van acumulando atomizados en el almacén por el efecto de la FHP.

Se puede apreciar que, con el ATP real y planificado reflejado en la Tabla I, es posible hacer las reservas necesarias y atender a las cinco propuestas de pedido recibidas, por lo que todas ellas se pueden comprometer.

Sin embargo, la asignación realizada se puede volver infactible, debido al efecto de la FHP, cuando se finaliza la fabricación de los lotes planificados y se clasifica el producto. En la siguiente tabla (Tabla II) se refleja dicha situación. En concreto, se puede apreciar que el lote L50, con $1510 \mathrm{~m}^{2}$ planificados para el periodo 2 , ha dado lugar finalmente a cuatro sublotes; T1C1L50, T1C2L50, T2C1L50 y T2C2L50, con $455,240,325$ y $490 \mathrm{~m}^{2}$ respectivamente.
TABLA II. ACTUALIZACIÓN DEL ATP AL FINALIZAR LA FABRICACIÓN DEL LOTE L50 Y SU IMPACTO EN LAS ASIGNACIONES REALIZADAS PREVIAMENTE.

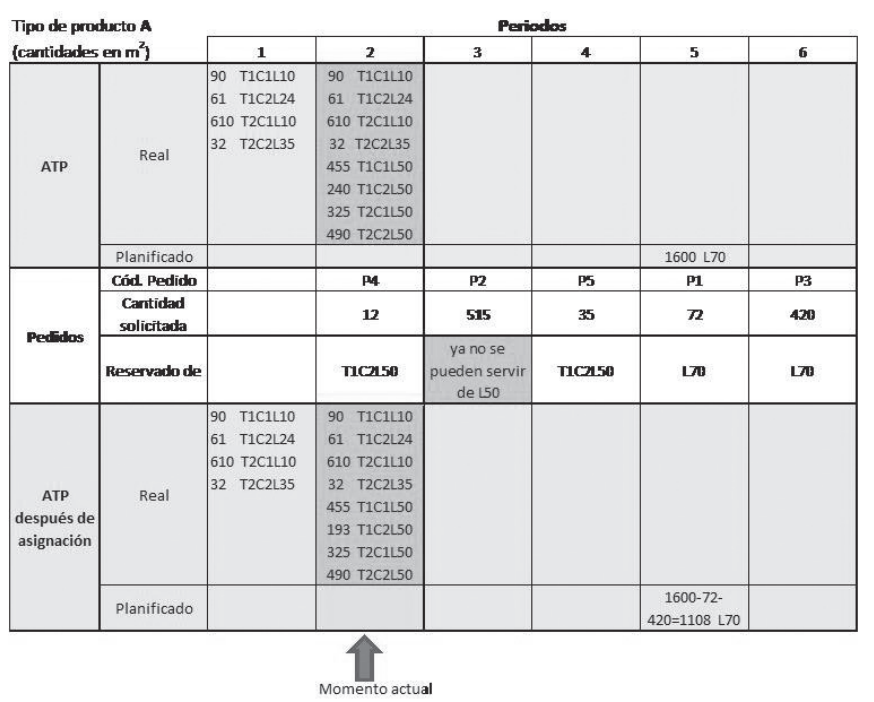

Esta atomización del lote L50 impide que se pueda servir el P2, ya que no hay una cantidad suficientemente grande del mismo tono y calibre para ello. Puesto que el P2 ya ha sido aceptado y comprometido con el cliente cuando se finaliza la fabricación del L50, la empresa deberá buscar ahora una solución. Una de las posibles actuaciones podría ser el lanzamiento a fabricación de nuevos lotes. Sin embargo, esta solución no puede asegurar una entrega a tiempo si el tiempo de suministro es mayor que la fecha de entrega del pedido. Además, esto podría suponer una interferencia a los lotes ya planificados y podría incrementar innecesariamente el inventario de la empresa y los costes. Se trata, por tanto, de buscar una opción que dote al sistema de la suficiente flexibilidad para adaptarse a una situación, que en contexto FHP, se dará habitualmente. Esta opción es la reasignación del inventario, que ahora será más complicada porque los sublotes del mismo tono y calibre son más pequeños y dan menos juego para encontrar soluciones.

En el caso recogido en la Tabla II, mediante el proceso de reasignación de inventario, se podría resolver el problema surgido utilizando los $610 \mathrm{~m}^{2}$ de ATP real del producto T2C1L10 para atender al pedido P2.

En definitiva, se deduce que la FHP ejerce un doble impacto en la reasignación del inventario, en este tipo de situaciones. Por una parte, provocando incertidumbre en las características finales del producto, impidiendo la consecución exacta de los planes, atomizando el inventario y generando, por todo ello, la necesidad de llevar a cabo una reasignación. Por otra parte, la FHP y la existencia de varios subconjuntos de productos con el mismo tono y calibre, pero no intercambiables ni acumulables, aumenta considerablemente la complejidad del proceso de reasignación del inventario.

\subsection{Impacto de la FHP en la reasignación del inventario cuando llegan propuestas de pedido prioritarias:}

La reasignación de pedidos puede resolver la situación provocada por la llegada de nuevas propuestas de pedido con alta prioridad que solicitan productos que ya han sido asignados previamente a otros pedidos con menor prioridad. La prioridad de propuestas de pedido recién llegadas puede 
ser alta debido a que provienen de un cliente más importante, que suponen un beneficio mayor para la empresa o que tienen una fecha de entrega más temprana. En estos casos parece razonable traspasar cantidades, ya comprometidas con pedidos de baja prioridad, para poder comprometer pedidos con una prioridad superior. De esta forma, los pedidos de menor prioridad, si tienen una fecha de entrega más tardía, podrán cumplimentarse con nuevos lotes cuya fabricación se planificará en periodos posteriores y, si suponen un menor beneficio para la empresa, podrán renegociarse con el cliente.

En situaciones de reasignación por la llegada de nuevos pedidos prioritarios, la FHP y su efecto de atomización del inventario, reducirá las posibles opciones de reasignación; es más fácil encontrar opciones de reasignar el inventario cuando este está formado por grandes cantidades de pocos subtipos de producto que cuando está formado por pequeñas cantidades de muchos subtipos de productos.

A continuación se incluye una tabla que recoge este tipo de situaciones. Teniendo en cuenta que ya se ha analizado el impacto de la FHP en el ATP planificado (en el apartado anterior), en este apartado sólo se utilizará ATP real para facilitar el entendimiento de la situación concreta que se pretende analizar. Así, en la Tabla III, se muestra el ATP real inicial del producto A para cada subtipo de producto, las propuestas de pedido según su orden de llegada y el ATP real resultante después de haber comprometido cada propuesta.

TABla III. ASIgNACión de ATP REAL SEgÚN POLÍticA FCFS.

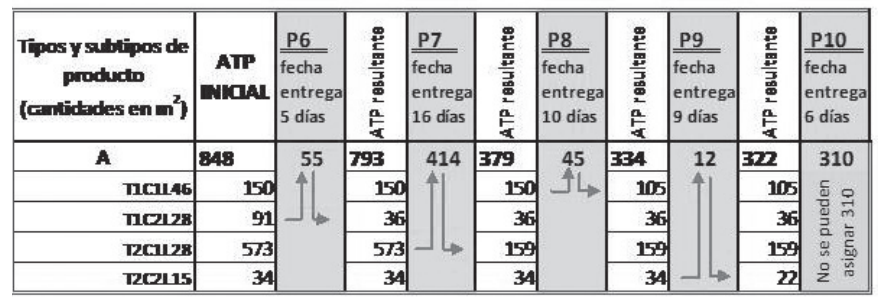

En Tabla III se reproduce la situación en la que llega un pedido (P10) con fecha de entrega temprana (6 días) que no se puede atender porque gran parte del ATP real inicial ya ha sido asignado a otros pedidos (P6, P7, P8 y P9) con fechas de entrega más tardías, pero que han llegado antes.

Obsérvese que, por el efecto de la FHP, el pedido P10 no se podrá servir porque no hay ATP real suficiente de ninguno de los subtipos del producto, a pesar de que el ATP resultante total antes de la llegada de P5 $\left(322 \mathrm{~m}^{2}\right)$ es mayor que lo que hace falta para P10 $\left(310 \mathrm{~m}^{2}\right)$. Es decir, si no existiera la FHP, el inventario no estaría atomizado, y por tanto, el ATP inicial acumulado sería de $848 \mathrm{~m}^{2}$ de A, sencillamente. Así, sería posible servir todos los pedidos, incluido P10, ya que la suma de $\mathrm{m}^{2}$ solicitados en los cinco pedidos $(55+414+45+12+310=836)$ es inferior al ATP inicial $\left(848 \mathrm{~m}^{2}\right)$.

En la Tabla III, también se puede apreciar que, la propuesta de pedido P6 podría servirse con los subtipos T1C2L46, T1C2L28 y T2C1L28 (del subtipo T2C2L15 no hay cantidad suficiente). En este caso, según la política de la empresa, se escoge el T1C2L28 porque deja un menor resto de producto.

Dada esta situación, parecería lógico realizar una reasignación mediante la cual se intentase traspasar ATP, desde los pedidos P7 o P8 con fecha de entrega más tardía, al pedido P10. En este caso, mediante la reasignación, se podrían recuperar el producto inicialmente reservado para el pedido P7 con fecha de entrega en 16 días, y utilizarlo para el pedido P10 con fecha de entrega mucho más temprana (6 días). Así se podría asegurar la entrega de los pedidos más urgentes (P10) y se ganaría tiempo para poder resolver la entrega de los pedidos menos urgentes (P7 o P8) y que ya no tendrían producto asignado porque lo han cedido a los más urgentes.

Por todo ello, se puede concluir que la FHP ejerce un claro impacto en la reasignación del inventario cuando llegan propuestas de pedido prioritarias, añadiendo una nueva restricción que cumplir en la reasignación (no servir a un mismo cliente con producto de distintos tonos y calibres) y complicando la búsqueda de soluciones por el efecto de la atomización del inventario.

\subsection{Impacto de la FHP en la reasignación del inventario en la situación cuando hay modificaciones por parte del cliente en los pedidos ya comprometidos:}

Ciertos cambios en los pedidos, por ejemplo, cuando el cliente solicita una nueva fecha de entrega, modifica la cantidad pedida o anula su pedido, pueden afectar de manera importante a las asignaciones previamente realizadas, especialmente en contextos de FHP. La factibilidad de este tipo de peticiones del cliente, en las que solicita una modificación de uno de sus pedidos previamente aceptado, deberá analizarse en cada caso, por parte de la empresa.

En la Tabla IV se ha reproducido la situación en la que se reciben cuatro pedidos (P6 a P9) y, después, llega una propuesta de modificación sobre uno de ellos (P8 con 45 $\left.\mathrm{m}^{2}\right)$, mediante la cual el cliente pretende aumentar en $25 \mathrm{~m}^{2}$ la cantidad inicialmente solicitada $\left(45 \mathrm{~m}^{2}\right)$. La propuesta de modificación se ha representado como la llegada de un nuevo pedido codificado como P8b, por valor de $25 \mathrm{~m}^{2}$. Del producto inicialmente reservado para el pedido P8 (T1C1L46) ya no quedan más que $15 \mathrm{~m}^{2}\left(60-45=15 \mathrm{~m}^{2}\right)$ así que, en principio, el nuevo pedido P8b debería ser rechazado porque no habría producto suficiente.

TABla IV. AsignACión de ATP REAL SEGÚN POLÍTICA FCFS.

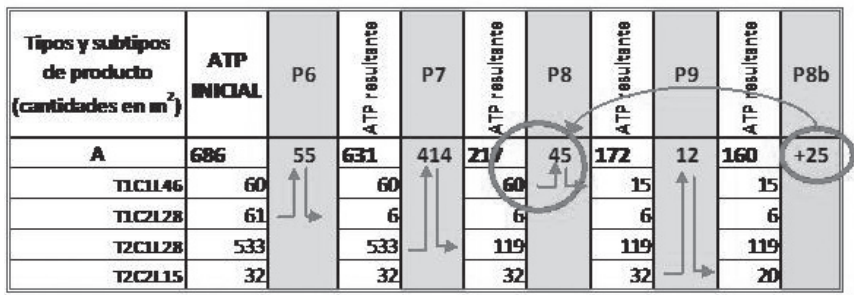

Normalmente, un incremento de la cantidad de producto solicitada en un pedido, supone para la empresa un aumento del beneficio obtenido en el pedido, por lo que será interesante para la empresa (y también para el cliente) encontrar la manera de aceptar dicha modificación.

En este sentido, gracias a la reasignación de inventario, es posible encontrar una solución para atender, tanto a la modificación del pedido P8 (P8b), como a los pedidos previamente comprometidos (Tabla V). En este caso, reasignando el producto T2C1L28, del que sí hay cantidad suficiente $\left(119 \mathrm{~m}^{2}\right)$, a los pedidos P8 $\left(45 \mathrm{~m}^{2}\right)$ y P8b $\left(25 \mathrm{~m}^{2}\right)$ que, agregados, formarían un nuevo pedido denominado P8' de $70 \mathrm{~m}^{2}$ (P8+P8b). 
TABLA V. REASIGNACIÓN DEL INVENTARIO PARA SOLUCIONAR EL PROBLEMA DE P8' SURGIDO AL AUMENTAR LA CANTIDAD SOLICITADA PREVIAMENTE EN P8.

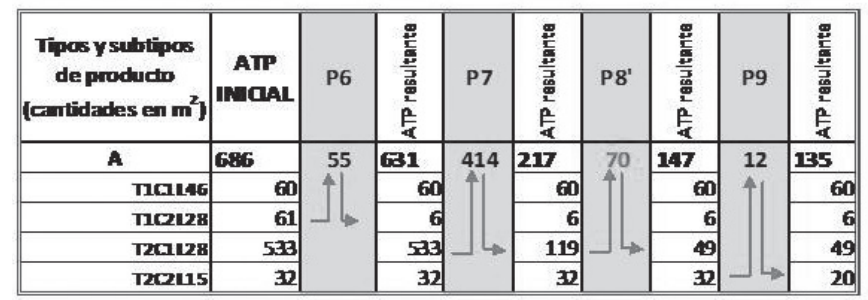

Por lo tanto, según el caso planteado, la FHP impacta en la reasignación del inventario en este tipo de situaciones de manera similar a cómo lo hace en la situación comentada en el apartado anterior: atomizando el inventario, añadiendo una nueva restricción a la reasignación y reduciendo las posibles soluciones.

\section{CONCLUSIONES}

La asignación de ATP a pedidos en contextos de fabricación MTS es de suma importancia: una asignación inicial adecuada puede influir en la satisfacción del cliente y en los beneficios de la empresa. Sin embargo, una asignación inicial adecuada puede pasar a ser inadecuada e incluso infactible. Para estos casos se ha justificado que, una posible solución, es la reasignación del inventario. La decisión de reasignación del inventario será más compleja cuanto más ambiciosos sean los objetivos que se quieran alcanzar, cuanto mayor sea el volumen de información a manejar y cuanto mayores sean las posibilidades de reasignación o combinaciones que se deban considerar.

En el presente trabajo, tras haber definido y formalizado el concepto de FHP, se ha descrito la problemática de la FHP en empresas cerámicas. Posteriormente, se han identificado las situaciones en las que una determinada asignación de ATP a pedidos puede dejar de ser adecuada y se ha propuesto la reasignación como una forma de búsqueda de nuevas asignaciones válidas. Se ha analizado el impacto de la FHP en cada una de las situaciones identificadas, observando que la FHP provoca una de éstas situaciones y complica, en todas ellas, la reasignación del inventario a pedidos. Para cada una de las situaciones de reasignación analizadas, se han presentado datos numéricos que permiten entender fácilmente cómo se produce la correspondiente situación y cómo influye la FHP en ella. Una de las principales aportaciones del presente trabajo es la descripción de la problemática de la FHP y su conexión con la gestión del ATP y la reasignación de inventario. Tras el análisis realizado, cabe concluir que la FHP provoca la atomización del inventario y aumenta considerablemente la complejidad de la reasignación de inventario, dificultando la obtención de soluciones factibles y adecuadas.

Para el caso de estudio presentado, sólo se han considerado datos de un tipo de producto, cuatro subtipos, cinco pedidos para cada situación y una sola línea por pedido, obtenidos a partir de la simplificación de los datos reales de una empresa del sector cerámico. Sin embargo, se debe considerar que en una situación real de reasignación de inventario a pedidos en una empresa cerámica, pueden haber cientos de tipos de productos, miles de subtipos y cientos, incluso miles de pedidos en cartera con varias líneas de pedido y cantidades solicitadas muy dispares que oscilan desde los quince o veinte $\mathrm{m}^{2}$ hasta miles de $\mathrm{m}^{2}$. Las incertidumbre asociada a la FHP implica la necesidad de reasignación de inventario a los pedidos $\mathrm{y}$, al mismo tiempo, añade complejidad a dicha reasignación debido al efecto de atomización del ATP, el cual complica la búsqueda de soluciones al elevar las posibles combinaciones a analizar. Más aún, cuando en situaciones reales, un pedido está compuesto por varias líneas de pedido y la no cumplimentación de una ellas significa la no cumplimentación del pedido completo. En estos casos, la decisión de reasignación de un tipo de producto a una línea de pedido afecta al pedido completo y consecuentemente, al resto de productos que se solicitan en el mismo. Para poder abordar esta compleja situación será necesaria la utilización de herramientas de ayuda a la toma de decisiones que permitan encontrar al decisor las mejores soluciones en el menor tiempo posible. Actualmente, las empresas cerámicas se suelen apoyar en herramientas basadas en hojas de cálculo. Sin embargo, debido a la gran cantidad de datos utilizados y las posibilidades de reasignación, se hace necesaria la utilización de herramientas más sofisticadas como modelos de programación matemática o modelos de simulación.

Por todo ello, actualmente se está trabajando en dos líneas de investigación. La primera contempla el desarrollo de herramientas de ayuda a la toma de decisiones basadas en el modelado matemático para la reasignación de inventario a pedidos multilínea en contextos de FHP. La segunda, estudia y documenta la problemática de la FHP y la reasignación del inventario en otro tipo de empresas distintas a las cerámicas.

\section{AGRADECIMIENTOS}

El presente trabajo se ha desarrollado parcialmente, tanto en el marco del proyecto de investigación financiado por el Ministerio de Educación y Ciencia español titulado "Potenciación de la competitividad del tejido empresarial español a través de la logística como factor estratégico en un entorno global" (PSE-370000-2008-8), como en el proyecto de investigación financiado por el Ministerio de Ciencia e Innovación español titulado "Personalización en Masa y Cadenas de Suministro Inteligentes, con Productos y Procesos Complejos (PERMACASI)" (DPI 2008-06788-C02-01), dentro de los cuales se ha tenido oportunidad de conocer y analizar la problemática expuesta en el presente trabajo en una empresa líder del sector cerámico español.

\section{REFERENCIAS}

1. M. M. E. Alemany, F. Alarcón, A. Ortiz, F. C. Lario y M. A. Bengochea, “El proceso de comprometer pedidos de un paquete de productos integrado por productos del sector cerámico y productos complementarios: Parte I Descripción y caracterización de la problemática.", Bol. Soc. Esp. Ceram., vol. 46 [1], págs. 21-28, (2007)

2. F. Alarcón, M. M. Alemany, A. Ortiz y F. C. Lario, "'Order promising" y conceptos asociados: revisión de significados e interpretaciones y propuesta para su clasificación", IX Congreso de Ingeniería de Organización, Gijón, 8-9 sept., (2005). 
3. B. Fleischmann y H. Meyr, "Customer orientation in advanced planning systems.", Springer, págs. 298-322, (2004).

4. A. M. Roma y J. M. Castan, “La cadena de suministro para empresas que en su proceso de producción incorporan materias primas procedentes directamente de la naturaleza", 3rd International Conference on Industrial Engineering and Industrial Management, Barcelona-Terrassa, 2-4 sept., págs. 1692-1700, (2009).

5. J. A. Heredia y M. Gras, "Análisis y modelado de la transmisión de variabilidad dimensional en un proceso de producción de baldosas cerámicas.", Bol. Soc. Esp. Ceram., vol. 48 [6], págs. 289-296, (2009).

6. M. O. Ball, C. Y. Chen y Z. Y. Zhao, "Available to Promise.", Kluwer Academic Publishers, págs. 447-483, (2004).

7. P. Keskinocak y S. Tayour, "Due Date Management Policies.", Modeling in the E-business Era, págs. 485-554, (2004).

8. R. Pibernik y P. Yadav, "Inventory reservation and real-time order promising in a Make-to-Stock system.", OR Spectrum, vol. 31 [1], págs. 281307, (2009).

9. R. Pibernik, "Advanced available-to-promise: Classification, selected methods and requirements for operations and inventory management.", Int. J. Prod. Econ., vol. 93-94 [1], págs. 239-252, (2005).

10. E. T. Kirche, S. N. Kadipasaoglu y B. M. Khumawala, “Maximizing supply chain profits with effective order management: integration of ActivityBased Costing and Theory of Constraints with mixed-integer modelling.", Int. J. Prod. Res., vol. 43 [7], págs. 1297-1311, (1-4-2005).

11. B. Watanapa y A. Techanitisawad, "Simultaneous price and due date settings for multiple customer classes.", Eur. J. Oper. Res., vol. 166 [2], págs. 351-368, (16-10-2005).

12. B. Jeong, S. B. Sim, H. S. Jeong y S. W. Kim, "An available-to-promise system for TFT LCD manufacturing in supply chain.", Comput. Ind. Eng., vol. 43 [1-2], págs. 191-212, (2002).

13. C. Y. Chen, Z. Zhao y M. O. Ball, "A Model for Batch Advanced AvailableTo-Promise.", Prod. Oper. Manag., vol. 11 [4], págs. 424-440, (2002).

14. D. M. Topkis, "Optimal Ordering and Rationing Policies in a Nonstationary Dynamic Inventory Model with n Demand Classes.", Manage. Sci., vol. 15 [3], págs. 160-176, (1968).

15. S. Benjaafar, M. ElHafsi y F. d. Véricourt, "Demand Allocation in MultipleProduct, Multiple-Facility, Make-to-Stock Systems.", Manage. Sci., vol. 50 [10], págs. 1431-1448, (2004).

16. R. Pibernik, "Managing stock-outs effectively with order fulfilment systems.", J. Manuf. Tech. Manag., vol. 17 [6], págs. 721-736, (2006).

17. H. Meyr, "Customer segmentation, allocation planning and order promising in make-to-stock production.", OR Spectrum, vol. 31 [1], págs. 229-256, (2009).
18. J. L. Amorós, A. Blasco, J. E. Enrique, V. Beltrán y A. Escardino, "Variables en la compactación de soportes cerámicos de pavimento y revestimento.", Técnica Cerámica, vol. 105, págs. 792-812, (1982).

19. J. L. Amorós, A. Blasco, V. Beltrán y F. Negre, "Pastas de gres de monococción. Influencia de las variables del proceso en la calidad del producto acabado.", Técnica Cerámica, vol. 120, págs. 1368-1384, (1984).

20. G. Mallol, D. Llorens, C. Felíu y F. Castro, "Medida en continuo de la humedad de los soportes cerámicos prensados.", Cerámica Información, vol. 289, págs. 81-90, (2002).

21. J. E. Hernández, E. Valencia y E. Villar, "Influencia de la humedad en el comportamiento de la resistencia a la compresión en mezclas de moldeo.", Bol. Soc. Esp. Ceram., vol. 40 [2], págs. 107-113, (2001).

22. C. Ferrer, D. Llorens, G. Mallol, E. Monfort y A. Moreno, “Optimización de las condiciones de funcionamiento en hornos monoestrato. Medida de gradientes transversales de temperatura.", Técnica Cerámica, vol. 227, págs. 653-662, (1994)

23. J. L. Amorós, V. Bagán, M. J. Orts y A. Escardino, “La operación de prensado en la fabricación de pavimentos por monococción. I. Influencia del polvo de prensas sobre las propiedades de las piezas en crudo.", Bol. Soc. Esp. Ceram., vol. 27 [5], págs. 273-282, (1988).

24. E. Vallada, C. Maroto, R. Ruiz y B. Segura, "Problemas de programación de la producción en el sector cerámico español", 27 Congreso Nacional de Estadística e Investigación Operativa, Lleida, 8-11 abril, págs. 638-676, (2003).

25. A. Poyatos, R. Bonaque, G. Mallol y J. Boix, “Nuevo sistema y metodología para la eliminación de los calibres en el proceso de fabricación de baldosas cerámicas.”, Bol.Soc.Esp.Ceram., vol. 49 [2], págs. 147-151, (2010).

26. E. Vallada, C. Maroto, R. Ruiz y B. Segura, “Análisis de la programación de la producción en el sector cerámico español.”, Bol. Soc. Esp. Ceram., vol. 44 [1], págs. 39-44, (2005).

27. I. Tortajada, G. Peris-Fajarnes, M. Aguilar y P. Latorre, "Análisis del proceso de clasificación cerámica.", Bol. Soc. Esp. Ceram., vol. 45 [1], págs. 22-27, (2006).

28. C. Boukouvalas, J. Kittler, R. Marik, M. Mirmehdi y M. Petrou, “Ceramic tile inspection for colour and structural defects.", University of Bristol, (1995).

29. S. Kukkonen, H. Kalviainen y J. Parkkinen, "Color features for quality control in ceramic tile industry.", Opt. Eng., vol. 40 [2], págs. 170-177, (2001).

30. R. Massen, "Clasificación óptica automática de azulejos porcelanados.", Cerámica Información, vol. 252, págs. 29-32, (2000).

31. R. Massen, "Aumento de la producción cerámica con sistemas de visión bivalentes: inspección y seguimiento del proceso automático con la misma tecnología", QUALICER 2004, págs. 3-6, (2004). 